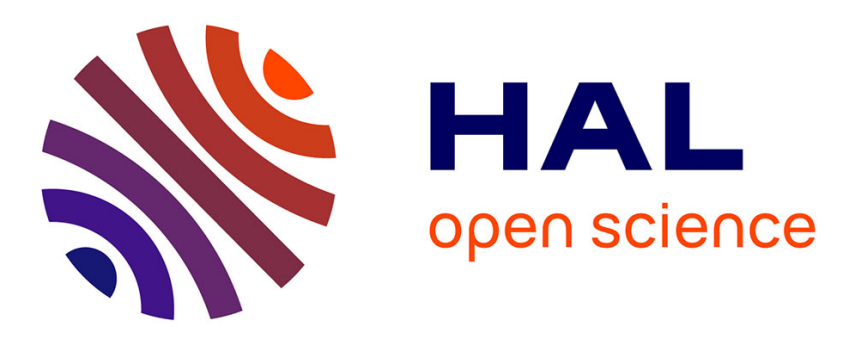

\title{
Full vertical car observer design methodology for suspension control applications
}

Luc Dugard, Olivier Sename, Sébastien Aubouet, Benjamin Talon

\section{To cite this version:}

Luc Dugard, Olivier Sename, Sébastien Aubouet, Benjamin Talon. Full vertical car observer design methodology for suspension control applications. Control Engineering Practice, 2012, 20 (9), pp.832845. 10.1016/j.conengprac.2012.04.008 . hal-00706698

\section{HAL Id: hal-00706698 https://hal.science/hal-00706698}

Submitted on 11 Jun 2012

HAL is a multi-disciplinary open access archive for the deposit and dissemination of scientific research documents, whether they are published or not. The documents may come from teaching and research institutions in France or abroad, or from public or private research centers.
L'archive ouverte pluridisciplinaire HAL, est destinée au dépôt et à la diffusion de documents scientifiques de niveau recherche, publiés ou non, émanant des établissements d'enseignement et de recherche français ou étrangers, des laboratoires publics ou privés. 


\title{
Full vertical car observer design methodology for suspension control applications
}

\author{
L. Dugard ${ }^{\mathrm{a}}$, O. Sename ${ }^{\mathrm{a}, 1}$, S. Aubouet ${ }^{\mathrm{b}}$, B. Talon $^{\mathrm{b}}$ \\ ${ }^{a}$ GIPSA-lab, Control Systems Dpt, UMR CNRS 5216, 11 rue des Mathématiques, BP46, 38402 \\ Saint-Martin d'Hères Cedex, FRANCE \\ ${ }^{b}$ SOBEN S.A.S., Pôle Mécanique d'Alès Cévennes, Vallon de Fontanes, 30520 St-Martin de Valgalgues, \\ FRANCE
}

\begin{abstract}
This paper deals with the design of an $\mathcal{H}_{\infty}$-observer to estimate the state variables of the vertical car dynamics to be used for suspension control applications. The proposed methodology allows to cope with both the disturbance decoupling problem (for the road profile effects) and the implementation issue (through observer pole placement). The considered model is a 7 DOF full-car vertical model subject to unknown ground disturbances whose effects on the estimated state variables are minimized using the $\mathcal{H}_{\infty}$ framework. Some experiments on a real test car highlight the performances of this observer which could be used in many advanced control strategies to improve the comfort and road holding of a vehicle using a reduced number of sensors.
\end{abstract}

Keywords: $\mathcal{H}_{\infty}$-observer, unknown input, Linear Matrix Inequality (LMI), suspension control, pole placement

pdf

\section{Introduction}

This paper deals with the estimation of some non-measured variables of the vehicle model, for suspension control objectives. In this section, the main objectives are first presented. Then, a brief state of the art, dealing both with existing observers for suspension

Email addresses: luc.dugard@gipsa-lab.fr (L. Dugard), olivier.sename@gipsa-lab.fr (O. Sename )

${ }^{1}$ Corresponding author. Tel.: +334768262 32 
control applications and unknown input observers in general, is provided. Finally, the contribution of the paper is presented.

\subsection{Problem statement}

The aim is to estimate the state variables of the full-car model in order to get a com-

plete knowledge of the vehicle dynamics for on-board suspension control applications. The developed observer has to provide the estimated state variables using a reduced number of sensors. This is one of the main challenges since many car or equipment manufacturers like SOBEN currently aim at equipping mass-produced cars with controlled suspensions to improve comfort and road holding performances. However, due to the number and the cost of the required sensors, this is not yet possible, except for upmarket car models. Moreover even if such cars have been already equipped with controlled suspensions, the control strategies are often open-loop since closed-loop applications require many sensors. Furthermore, car manufacturers need to choose the number, the kind and the location of sensors in the vehicle. Therefore, a complete observer design methodology is of great interest to take up this challenge and meet the industrial needs.

\subsection{State of the art}

Observers for suspension control applications have been already studied (?????). In these previous works, bi-linear observers, also based on acceleration measurements, are proposed and provide interesting results, but for quarter car model estimation only. In ?, the necessary and sufficient conditions for bi-linear observer design are established. However, if these restrictive conditions for exact disturbance decoupling are not fulfilled, no result is proposed to design an approximated observer. Furthermore the location of the observer poles cannot be explicitly specified, which renders the proposed observers quite difficult to adjust in practice. In these past studies, the estimation problem is often addressed using specific sensors, and the developed methodology sometimes cannot be adapted if some other variables are measured. Furthermore, the proposed methods often do not include tuning parameters allowing the designer to easily adapt the observer behavior to the observed system. Therefore, no global and practical methodology exists to solve this problem. 
The system under study is represented by a full-car model, subject to unknown ground disturbances. Thereafter, unknown input observers (UIO) have to be considered. Such observers have been already studied by many authors ??????. Various methods have been proposed to obtain an exact disturbance decoupling under specific conditions, or to minimize the disturbance effect on the estimated states (?). In ?, a method is proposed to design an UIO where the measured variables are also corrupted by the unknown disturbance, through some linear transformations that allow the cancellation of the disturbance effect on the measured outputs. Depending on the system and on the available measurements, the conditions for exact disturbance decoupling, given for example in ?? may not be fulfilled. These mathematical conditions are necessary to get an exact disturbance decoupled observer, but in practice, they are not necessary to get an efficient observer since they involve an exact decoupling on the whole frequency range. Indeed, from a practical point of view, these conditions should be achieved on the frequency range of interest only. Furthermore, the exact disturbance decoupling may be tractable and might achieve unsuitable pole placement, leading to unusable observer. However, the tradeoff between disturbance decoupling and observer pole placement has not been studied sufficiently thoroughly.

\subsection{Contribution}

The main contribution is a complete observer design methodology, based on existing results on UIO design, and moreover taking some practical implementation constraints into account, such as pole placement or $\mathcal{H}_{\infty}$ disturbance decoupling. The unknown disturbance effect minimization problem is formulated such that the observer matrices are determined to fulfill a global condition including all the essential objectives: stability, disturbance decoupling and pole placement, without any starting choice in the observer matrices, like in many existing studies. Therefore this method is less conservative and allows the designer to handle the compromise between disturbance decoupling and pole placement, thanks to appropriate tuning parameters and LMI ( Linear Matrix Inequality) regions for pole placement.

The proposed methodology leads to the design of an $\mathcal{H}_{\infty}$-observer to estimate the state variables of the vertical car dynamics. This kind of observer can be used for any 
passive suspension system (for instance for diagnosis purposes), but is very interesting for semi-active suspension systems, since it could be used in different observer-based control strategies. It is worth noting that, even if the observer is a LTI system, it is proven to be efficient with respect to the variation of the damping coefficient of the suspension, which, in practice, corresponds to different control actions (for the semi-active damper).

This paper is organized as follows: the material used for the experiments and the vehicle model are described in Section 2, the estimation problem is formulated and solved in Section 3, some synthesis results are given and the robustness of the observer is analyzed in Section 4, the experimental tests and results are presented in Section 5. Finally, the paper is concluded in Section 6, and some future works are proposed.

$$
\text { pdf }
$$

\section{Vehicle modeling and system description}

pdf In this section, the various material resources used for the experiments shown in this paper to analyze the behavior of the synthesized observer are presented.

\subsection{Material resources}

\subsubsection{Semi-active shock absorber prototypes}

Four semi active shock absorber prototypes, under study in this paper, have been built by SOBEN and mounted on a test car. These shock absorbers can be controlled by means of a servomechanism installed on each shock absorber to control the internal oil flow, and therefore, the damping properties. The Figure 1 represents a front shock absorber prototype mounted on the SOBEN test car.

Figure 1: New semi-active SOBEN shock absorber

\subsubsection{Test car and acquisition/control board}

The test car from SOBEN is a mid-range car used for the experiments. Several sensors

have been mounted on the test car, as represented in Figure 1. Some of them, like the 
accelerometers are used by the observer, whereas the others have been installed only to study the behavior of the suspensions and to validate the estimated variables, as detailed in Section 4. Seven accelerometers have been mounted. The four vertical unsprung masses accelerations $\ddot{z}_{u s_{i=1,2,3,4}}$, at each corner of the vehicle, the vertical sprung mass accelerations $\ddot{z}_{s_{1,2,3}}$ at three corners, and the four suspension deflections are measured.

Notice that the last sprung mass acceleration measurement is not required since the vehicle body is supposed to be a solid having three degrees of freedom. Indeed, each acceleration of the sprung mass at one corner can be simply derived from the accelerations of the sprung mass at the other three corners.

The accelerometers are strain-gage based, whereas the deflection sensors are potentiometer based. They have been chosen because of their reduced cost, high reliability and small packaging. The measured variables and used sensors are given in Table 1.

\begin{tabular}{clc}
\hline \multicolumn{1}{c}{ Notation } & Description & Full scale \\
\hline \hline$\ddot{z}_{u s_{1}}$ & Front left wheel vertical acceleration & $+/-500 \mathrm{~m} / \mathrm{s}^{2}$ \\
$\ddot{z}_{u s_{2}}$ & Front right wheel vertical acceleration & $+/-500 \mathrm{~m} / \mathrm{s}^{2}$ \\
$\ddot{z}_{u s_{3}}$ & Rear left wheel vertical acceleration & $+/-500 \mathrm{~m} / \mathrm{s}^{2}$ \\
$\ddot{z}_{u s_{4}}$ & Rear left wheel vertical acceleration & $+/-500 \mathrm{~m} / \mathrm{s}^{2}$ \\
$\ddot{z}_{s_{1}}$ & Front left body vertical acceleration & $+/-50 \mathrm{~m} / \mathrm{s}^{2}$ \\
$\ddot{z}_{s_{2}}$ & Front right body vertical acceleration & $+/-50 \mathrm{~m} / \mathrm{s}^{2}$ \\
$\ddot{z}_{s_{3}}$ & Rear left body vertical acceleration & $+/-50 \mathrm{~m} / \mathrm{s}^{2}$ \\
$z_{d e f_{1}}=z_{s_{1}}-z_{u s_{1}}$ & Front left suspension deflection & $0-0.2 \mathrm{~m}$ \\
$z_{d e f_{2}}=z_{s_{2}}-z_{u s_{2}}$ & Front right suspension deflection & $0-0.2 \mathrm{~m}$ \\
$z_{d e f_{3}}=z_{s_{3}}-z_{u s_{3}}$ & Rear left suspension deflection & $0-0.2 \mathrm{~m}$ \\
$z_{d e f_{4}}=z_{s_{4}}-z_{u s_{4}}$ & Rear right suspension deflection & $0-0.2 \mathrm{~m}$ \\
$F_{i}, i=1, . ., 4$ & Shock absorber forces & Confidential \\
\hline
\end{tabular}

Table 1: Test car: measured variables and sensors

The complete acquisition and control board/arcitecture is detailed in the appendix. 


\subsection{Vertical full-car model}

This section aims at presenting the well-known vertical full-car model. This simplified models describes the main dynamics of the vehicle and can be used for control or estimation purposes. The full vertical car model, firstly described in ??, and represented in Figure 2, is made up with a sprung mass in vertical translation, and rotating on two horizontal axes, and the four unsprung masses, each one in vertical translation. Each unsprung mass is linked to the ground with a tire modeled by a stiffness, and to the sprung mass with a suspension made up with a linear shock absorber and a linear spring. The various forces acting on sprung $\left(m_{s}\right)$ and unsprung $\left(m_{u s_{i}} ; i=1,4\right)$ masses are the usual ones (given by equation 2). This model is classical and has already been used in ??? The variables and parameters of this model are given in Table 2.

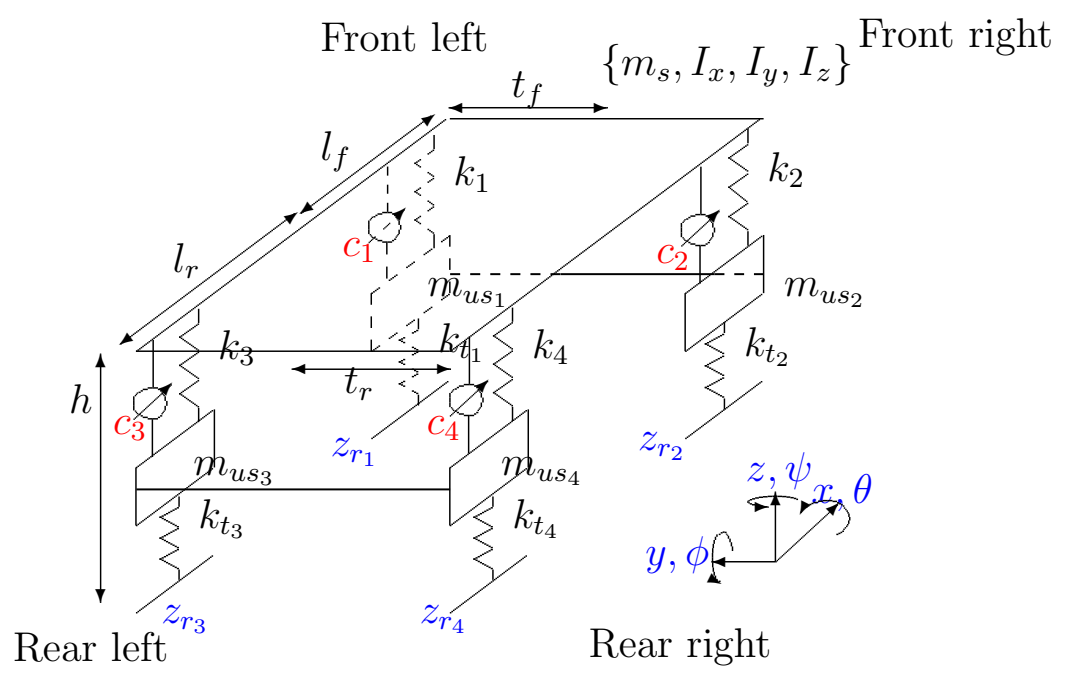

Figure 2: Vertical full-car model with 14 DOF

The vertical full-car model presented in Figure 2 is governed by the equations of motion 


\begin{tabular}{|c|c|c|}
\hline Parameter/variable & Description & Value \\
\hline$m_{s}$ & Sprung mass & $1450 \mathrm{~kg}$ \\
\hline$m_{u s_{1,2}}$ & Front unsprung masses & $39 \mathrm{~kg}$ \\
\hline$m_{u s_{3,4}}$ & Rear unsprung masses & $32 \mathrm{~kg}$ \\
\hline$k_{1,2}$ & Front suspension stiffness & $30000 \mathrm{~N} / \mathrm{m}$ \\
\hline$k_{3,4}$ & Rear suspension stiffness & $18000 \mathrm{~N} / \mathrm{m}$ \\
\hline$c_{1,2}$ & Front linear damping rate & $4000 \mathrm{Ns} / \mathrm{m}$ \\
\hline$c_{3,4}$ & Rear linear damping rate & $3000 \mathrm{Ns} / \mathrm{m}$ \\
\hline$k_{t 1,2,3,4}$ & Tire stiffness & $200000 \mathrm{~N} / \mathrm{m}$ \\
\hline$I_{x}, I_{y}$ & Roll and pitch inertia & $610,2750 \mathrm{~kg} \cdot \mathrm{m}^{2}$ \\
\hline$t_{f}$ & Distance $\mathrm{COG}^{1}$ - front left tire & $0.75 \mathrm{~m}$ \\
\hline$t_{r}$ & Distance COG - rear left tire & $0.75 \mathrm{~m}$ \\
\hline$l_{f}$ & Distance COG - front & $1.06 \mathrm{~m}$ \\
\hline$l_{r}$ & Distance COG - rear & $1.7 \mathrm{~m}$ \\
\hline$z_{r_{i}}$ & Ground vertical positions & $i=1 . .4$ \\
\hline$z_{s}$ & COG sprung mass position & \\
\hline$z_{s_{i}}$ & Sprung mass positions & $i=1 . .4$ \\
\hline$z_{u s_{i}}$ & Unsprung mass positions & $i=1 . .4$ \\
\hline$F_{s_{i}}$ & Suspension forces & $i=1 . .4$ \\
\hline$\theta$ & Sprung mass roll angle & \\
\hline$\phi$ & Sprung mass pitch angle & \\
\hline$F_{z}$ & Vertical disturbance force & \\
\hline$M_{x}, M_{y}$ & Disturbance moments & \\
\hline
\end{tabular}

${ }^{1}$ Center Of Gravity

Table 2: Vertical full-car parameters and variables 


$$
\left\{\begin{array}{l}
m_{s} \ddot{z}_{s_{s}}=-\left(F_{s_{1}}+F_{s_{2}}+F_{s_{3}}+F_{s_{4}}+F_{z}\right) \\
m_{u s_{1}} \ddot{z}_{u s_{1}}=\left(F_{s_{1}}-F_{t_{1}}\right) \\
m_{u s_{2}} \ddot{z}_{u s_{2}}=\left(F_{s_{2}}-F_{t_{2}}\right) \\
m_{u s_{3}} \ddot{z}_{u s_{3}}=\left(F_{s_{3}}-F_{t_{3}}\right) \\
m_{u s_{4}} \ddot{z}_{u s_{4}}=\left(F_{s_{4}}-F_{t_{4}}\right) \\
I_{x} \ddot{\theta}=\left(F_{s_{1}}-F_{s_{2}}\right) t_{f}+\left(F_{s_{3}}-F_{s_{4}}\right) t_{r}+M_{x} \\
I_{y} \ddot{\phi}=\left(F_{s_{4}}+F_{s_{3}}\right) l_{r}-\left(F_{s_{2}}+F_{s_{1}}\right) l_{f}+M_{y}
\end{array}\right.
$$

where the forces $F_{s i}$ and $F_{t i}$, respectively provided by the suspension $i$ and the tire $i$, are given by

$$
\left\{\begin{array}{l}
F_{s i}=k_{i} \cdot\left(z_{s_{i}}-z_{u s_{i}}\right)+c_{i} \cdot\left(\dot{z}_{s_{i}}-\dot{z}_{u s_{i}}\right) \\
F_{t i}=k_{t_{i}} \cdot\left(z_{u s_{i}}-z_{r_{i}}\right) .
\end{array}\right.
$$

Note that the sprung mass positions at each corner of the vehicle can be easily derived from the vehicle equations of motions, according to

$$
\left\{\begin{array}{l}
z_{s_{1}}=z_{s}+l_{f} \sin (\phi)-t_{f} \sin (\theta) \\
z_{s_{2}}=z_{s}+l_{f} \sin (\phi)+t_{f} \sin (\theta) \\
z_{s_{3}}=z_{s}-l_{r} \sin (\phi)-t_{r} \sin (\theta) \\
z_{s_{4}}=z_{s}-l_{r} \sin (\phi)+t_{r} \sin (\theta) .
\end{array}\right.
$$

These nonlinear equalities can be linearized as

$$
\left\{\begin{array}{l}
z_{s_{1}} \approx z_{s}+l_{f} \phi-t_{f} \theta \\
z_{s_{2}} \approx z_{s}+l_{f} \phi+t_{f} \theta \\
z_{s_{3}} \approx z_{s}-l_{r} \phi-t_{r} \theta \\
z_{s_{4}} \approx z_{s}-l_{r} \phi+t_{r} \theta
\end{array}\right.
$$

when $\theta$ and $\phi$ are small. In Section 3, the linear vertical full-car model (1) is used to design an observer.

pdf 


\section{Observer design}

pdf The main objective of this section is to propose a methodology to design observers for systems undergoing unknown disturbances. It is worth mentioning that the considered approach, inspired by the formulation proposed in ?, is very general and can be applied to a wide class of systems. In this section, this method is applied to estimate the unmeasured state variables of a vehicle model. This section is organized as follows : the estimation problem is formulated in Section 3.1. Then the disturbance decoupling conditions are studied in Section 3.2, the proposed observer is designed in Section 3.3, a pole placement method is given in Section 3.4, and finally, the complete design methodology is summarized in Section 3.5.

\subsection{General problem statement}

The full-car model (1), representing the system under study, is linear with 14 state variables and 7 inputs. This model can be written as a state-space model according to

$$
V(s)\left\{\begin{array}{l}
\dot{x}=A \cdot x+D_{x} \cdot v \\
y=C \cdot x+D_{y} \cdot v
\end{array}\right.
$$

where $x$ is the state vector, $v$ the input, $y$ the measured variables and $A \in \mathbb{R}^{n \times n}$, $D_{x} \in \mathbb{R}^{n \times d}, C \in \mathbb{R}^{m \times n}$ and $D_{y} \in \mathbb{R}^{m \times d}$. For the considered system, the state variables, the inputs and the 7 measurements used to estimate the full-car model are given by

$$
\left\{\begin{array}{l}
x=\left(\dot{z}_{s}, z_{s}, \dot{z}_{u s_{1}}, z_{u s_{1}}, \dot{z}_{u s_{2}}, z_{u s_{2}}, \dot{z}_{u s_{3}}, z_{u s_{3}}, \dot{z}_{u s_{4}}, z_{u s_{4}}, \dot{\theta}, \theta, \dot{\phi}, \phi\right) \\
v=\left(z_{r_{1}}, z_{r_{2}}, z_{r_{3}}, z_{r_{4}}, F_{z}, M_{x}, M_{y}\right) \\
y=\left(\ddot{z}_{s_{1}}, \ddot{z}_{s_{2}}, \ddot{z}_{s_{3}}, \ddot{z}_{u s_{1}}, \ddot{z}_{u s_{2}}, \ddot{z}_{u s_{3}}, \ddot{z}_{u s_{4}}\right) .
\end{array}\right.
$$

In the synthesis of the observer, the inputs $F_{z}, M_{x}$ and $M_{y}$ will be neglected. Indeed, $F_{z}$, $M_{x}$ and $M_{y}$ correspond to aerodynamic forces and load transfers. These disturbances are slow, and furthermore they are indirectly measured through the accelerometers mounted on the sprung mass. Therefore, it is not useful to consider them as unknown disturbances in the observer synthesis. Then $v$ becomes $v=\left(z_{r_{1}}, z_{r_{2}}, z_{r_{3}}, z_{r_{4}}\right)$ and only the ground disturbance effects are considered, which is clearly the actual unknown input in suspension systems. Furthermore, in the Equation (5), no control input is considered. This choice 
is explained in the next sections. However, all the results given in this section can be adapted if such an input is to be taken into account, as usual, in observer design.

The structure of the full-order observer chosen to estimate this model is given by

$$
\left\{\begin{array}{l}
\dot{z}=N \cdot z+L \cdot y \\
\hat{x}=z-E \cdot y
\end{array}\right.
$$

where $z \in \mathbb{R}^{n \times n}$ is the state variable of the observer and $\hat{x} \in \mathbb{R}^{n \times n}$ the estimated state variables. $N \in \mathbb{R}^{n \times n}, L \in \mathbb{R}^{n \times m}, E \in \mathbb{R}^{n \times m}$ are the observer matrices to be designed.

Let us define the matrices $P=\mathcal{I}_{n}+E C$ and $K=L+N E$. Then from the considered system (5) and the chosen observer structure (6), the estimation error dynamical equation is derived according to

$$
\begin{aligned}
\dot{e} & =\dot{x}-\dot{\hat{x}} \\
& =A x+D_{x} v-\dot{z}+E \dot{y} \\
& =(A-L C+E C A) x+\left(D_{x}-L D_{y}-N E D_{y}+E C D_{x}\right) v+E D_{y}-N(P x-e) \\
& =N e+(A-L C+E C A-N P) x+\left(P D_{x}-(L+N E) D_{y}\right) v+E D_{y} \dot{v} \\
& =N e+(P A-K C-N) x+\left(P D_{x}-K D_{y}\right) v+E D_{y} \dot{v} .
\end{aligned}
$$

The estimated state variable $\hat{x}$, described by (7), converges asymptotically to the state $x$ for any bounded initial conditions $\hat{x}(0)$ and $x(0)$ if and only if (??)

\section{Stability:}

$$
\left\{\begin{array}{l}
N \text { is Hurwitz } \\
N=P A-K C
\end{array}\right.
$$

\section{Disturbance decoupling:}

$$
\left\{\begin{array}{l}
K D_{y}-P D_{x}=(L+N E) D_{y}-P D_{x}=L D_{y}-P D_{x}+N E D_{y}=L D_{y}-P D_{x}=0 \\
E D_{y}=0
\end{array}\right.
$$

The observer design involves the calculation of the matrices $N, L$ and $E$ satisfying both the stability and disturbance decoupling conditions (8-9). However these conditions are not sufficient from a practical point of view. Indeed, the real-time implementation of the observer may not be possible if the poles are either too fast or too close to the 
imaginary axis. Therefore the eigenvalues of $N$ have to be placed in a well-chosen region fitting both the system bandwidth and the measurement noise level. These are the two main challenges in designing an efficient and implementable unknown input observer. A methodology to solve this problem is proposed in the next sections.

Definition 3.1 (Exact and $\mathcal{H}_{\infty}$-observers). A full state observer in the form of (6) is said to be,

- an exact observer if $N, L, E$ are exact solutions of (8) and (9). In this case, an exact disturbance decoupling is achieved since the estimated variables are not affected by the disturbance.

- an $\mathcal{H}_{\infty}$-observer if $N, L, E$ are obtained by minimizing the disturbance effect on the estimated state variables, i.e. the problem (10) has been solved,

$$
\min \gamma_{\infty} \text { s.t. }\|e\|_{2}<\gamma_{\infty} \cdot\|v\|_{2}
$$

where $\|.\|_{2}$ denotes the $\mathcal{L}_{2}$-norm. This norm represents the energy-to-energy gain of the considered system.

\subsection{Exact observer existence conditions}

Necessary and sufficient conditions for exact observer design are recalled (??), and a methodology to compute the observer matrices is given in this case.

\section{Stability conditions:}

The stability conditions (8) are fulfilled if and only if $(P A, C)$ is detectable. However, this condition depends on the matrix $P$. Therefore the stability conditions depend on the disturbance decoupling (9), as detailed below.

\section{Disturbance decoupling:}

Depending on the measurements, two cases have to be considered: 
- Case $D_{y}=\mathcal{O}$ :

In this case, the system (7) reduces to

$$
\dot{e}=N e-(N-P A+K C) x+P D_{x} v
$$

and the disturbance decoupling condition for the exact observer design becomes

$$
P D_{x}=0 \Leftrightarrow E C D_{x}=-D_{x}
$$

since $P=\mathcal{I}_{n}+E C$ by definition. This equation, where $E$ is the unknown, is solvable (?) if and only if

$$
\operatorname{rank}\left(C D_{x}\right)=\operatorname{rank}\left(\begin{array}{c}
C D_{x} \\
D_{x}
\end{array}\right),
$$

and there exists (?) an exact solution set fulfilling (12), in the form of

$$
E=-D_{x}\left(C D_{x}\right)^{+}+Y_{E}\left[\mathcal{I}_{m}-\left(C D_{x}\right)\left(C D_{x}\right)^{+}\right]
$$

where $Y_{E}$ is a free matrix of appropriate dimension.

- If $E$ can be chosen according to (14) in such a way that $(P A, C)$ is observable, the poles of $N$ can be arbitrarily assigned by choosing $K$ in the equation $N=P A-K C$. Then the last unknown matrix $L$ can be easily derived from $K=L+N E$, leading to an exact observer according to the Definition 3.1.

- If $E$ can be chosen according to (14) in such a way that $(P A, C)$ is detectable but not observable, some of the poles cannot be placed arbitrarily. Then $K$ can be computed to place the observable poles, and $L$ can be derived from $K=L+N E$, leading to an exact observer according to the Definition 3.1, However, if some of the non observable poles are too close to the imaginary axis, or too high, the disturbance decoupling will be exact, but the observer will not be implementable. In this case, an approximated observer, according to the Definition 3.1, has to be found with the best possible disturbance decoupling and implementable poles. A method to solve this problem is proposed in Section 3.3. 
- Case $D_{y} \neq \mathcal{O}$ :

In this case the disturbance decoupling conditions are given by

$$
\left\{\begin{array}{l}
L D_{y}-P D_{x}=0 \\
E D_{y}=0
\end{array}\right.
$$

In order to achieve an exact disturbance decoupling, $E$ has to fulfill $E D_{y}=0$. Since $D_{y}$ has full column rank, this equation, where $E$ is the unknown, can be solved and the solution set (?) is given by

$$
E=Y_{E}\left[\mathcal{I}_{m}-D_{y} D_{y}^{+}\right]
$$

where $Y_{E} \in \mathbb{R}^{n \times m}$ is a free matrix.

The other condition for exact disturbance decoupling is

$$
\begin{aligned}
& L D_{y}-P D_{x}=0 \\
\Leftrightarrow & L D_{y}=\left(\mathcal{I}_{n}+E C\right) D_{x} \\
\Leftrightarrow & L D_{y}=\left(\mathcal{I}_{n}+Y_{E}\left[\mathcal{I}_{m}-D_{y} D_{y}^{+}\right] C\right) D_{x} \\
\Leftrightarrow & L D_{y}-Y_{E}\left[\mathcal{I}_{m}-D_{y} D_{y}^{+}\right] C D_{x}=D_{x}
\end{aligned}
$$

which can also be parameterized as

$$
U_{L Y_{E}} \cdot \Psi_{L Y_{E}}=D_{x}
$$

where $U_{L Y_{E}}=\left[\begin{array}{ll}L & Y_{E}\end{array}\right]$ and $\Psi_{L Y_{E}}=\left[\begin{array}{c}D_{y} \\ -\left[\mathcal{I}_{m}-D_{y} D_{y}^{+}\right] C D_{x}\end{array}\right]$.

The Equation (18), where $U_{L Y_{E}}$ is the unknown, can be solved if and only if

$$
\operatorname{rank}\left(\Psi_{L Y_{E}}\right)=\operatorname{rank}\left(\begin{array}{c}
\Psi_{L Y_{E}} \\
D_{x}
\end{array}\right)
$$

and the solutions of (18) can be chosen among the family

$$
U_{L Y_{E}}=D_{x} \Psi_{L Y_{E}}^{+}+Y_{L Y_{E}}\left[\mathcal{I}_{n}-\Psi_{L Y_{E}} \Psi_{L Y_{E}}^{+}\right]
$$

where $Y_{L Y_{E}} \in \mathbb{R}^{n \times 2 * m}$ is a free matrix.

This parametrization enables us to determine both $E$ and $L$ such that the disturbance decoupling is perfect. However, the obtained solution set influences: 
- the detectability of $(P A, C)$, since $P=\mathcal{I}_{n}+E C$,

- the choice of $K$, allowing to place the poles of $P A-K C$, since $K$ depends on $E$ and $L$.

Therefore the disturbance decoupling conditions (15) reduce the solution set through a more restrictive detectability condition.

Furthermore, the stability condition $N=P A-K C$, with $K=L+N E$, has to be fulfilled. This condition can also be formulated as

$$
\begin{aligned}
N=P A-K C & \Leftrightarrow N=P A-L C-N E C \\
& \Leftrightarrow N P=P A-L C .
\end{aligned}
$$

This equation, where $N$ is the unknown, can be solved (?) if and only if

$$
\operatorname{rank}(P)=\operatorname{rank}\left(\begin{array}{c}
P A-L C \\
P
\end{array}\right)
$$

and the solutions of (21) are in the form of

$$
N=(P A-L C) P^{+}+Y_{N}\left(\mathcal{I}_{n}-P P^{+}\right)
$$

where $P=\mathcal{I}_{n}+E C$, and $Y_{N} \in \mathbb{R}^{n \times n}$ is a free matrix.

In (23), the matrices $L$ and $E$ are given by (20) and depend on $Y_{L Y_{E}}$. Therefore the choice of $Y_{L Y_{E}}$ influences the detectability of the pair $\left((P A-L C) P^{+},\left(\mathcal{I}_{n}-P P^{+}\right)\right)$. If the rank conditions (19) and (22) are fulfilled and if $\left((P A-L C) P^{+},\left(\mathcal{I}_{n}-P P^{+}\right)\right)$ is detectable, an exact observer can be determined and the observable poles cannot be chosen arbitrarily using $Y_{N}$.

If the rank conditions (19) and (22) are fulfilled and if $\left((P A-L C) P^{+},\left(\mathcal{I}_{n}-P P^{+}\right)\right)$ is observable, an exact observer can be determined and the poles can be arbitrarily chosen using $Y_{N}$.

If the rank conditions (19) and (22) are not fulfilled, the exact observer cannot be computed. 
Remark 3.1. Choosing $Y_{L Y_{E}}$ such that $\left((P A-L C) P^{+},\left(\mathcal{I}_{n}-P P^{+}\right)\right)$is observable, is not an easy problem, and may not be solved. Furthermore both (19) and (22) may not be fulfilled, and then no exact observer design is possible. However, an efficient approximated observer may exist and a global design methodology has to be established to help the designer find an optimal observer fulfilling all the theoretical conditions. Thereafter the solution consists in solving the whole problem including all the constraints. Such a method is proposed in Section 3.3.

\section{3. $\mathcal{H}_{\infty}$-observer design}

The system (7) will be parameterized such that the unknown observer matrices can be computed to minimize the disturbance effect on the estimated state variables. This parametrization is inspired by the formulation proposed in ?. The disturbance effect minimization is achieved by minimizing the $\mathcal{H}_{\infty}$-norm of the transfer from the unknown disturbance to the estimated state variables, which corresponds to the problem (10) given in the Definition 3.1.

The estimation error is governed by

$$
\dot{e}=N e+(P A-K C-N) x+\left(P D_{x}-K D_{y}\right) v+E D_{y} \dot{v} .
$$

This system is corrupted by the disturbance $v$ and its derivative $\dot{v}$, and can be rewritten as

$$
\dot{e}=N e+(P A-K C-N) x+F d
$$

where $F=\left(\begin{array}{cc}P D_{x}-K D_{y} & E D_{y}\end{array}\right)$ and $d=\left(\begin{array}{c}v \\ \dot{v}\end{array}\right)$.

Then the disturbance $F$ can be minimized according to the procedure described in the next sections. However, since $\dot{v}$ may be very high, $v$ may be less minimized. This method is possible but leads to a less efficient disturbance decoupling.

In order to avoid this problem, the observer matrix $E$ can be chosen according to (16). Therefore $E=Y_{E}\left[\mathcal{I}_{m}-D_{y} D_{y}^{+}\right]$, where the matrix $Y_{E}$ will be determined later, during the 
synthesis of the observer. Thereafter, $E D_{y}=\mathcal{O}$, and the system (24) can be rewritten as

$$
\dot{e}=N e+\left(P\left(Y_{E}\right) A-K C-N\right) x+\left(P\left(Y_{E}\right) D_{x}-L D_{y}\right) v
$$

where $P\left(Y_{E}\right)=\mathcal{I}_{n}+Y_{E}\left[\mathcal{I}_{m}-D_{y} D_{y}^{+}\right] C$.

The matrix $\Omega=\left[N, P, K, Y_{E}\right]$ is defined in order to parameterize the previous system. The equivalence

$$
\left\{\begin{array}{l}
P A-K C-N=\mathcal{O}_{n} \\
P-Y_{E}\left(\mathcal{I}_{m}-D_{y} D_{y}^{+}\right) C=\mathcal{I}_{n}
\end{array} \Leftrightarrow \Omega \cdot \Theta=\Psi\right.
$$

is first established, where $\Theta=\left[\begin{array}{rr}-\mathcal{I}_{n} & \mathcal{O}_{n} \\ A & \mathcal{I}_{n} \\ -C & \mathcal{O}_{m, n} \\ \mathcal{O}_{m, n} & -\left(\mathcal{I}_{m}-D_{y} D_{y}^{+}\right) C\end{array}\right], \Psi=\left[\mathcal{O}_{n}, \mathcal{I}_{n}\right]$, and $\mathcal{O}_{n}$ denotes the null matrix of size $n \times n$.

The equation $\Omega \cdot \Theta=\Psi$, where $\Omega$ is the unknown, can be solved if and only if

$$
\operatorname{rank}(\Theta)=\operatorname{rank}\left(\begin{array}{c}
\Theta \\
\Psi
\end{array}\right)
$$

holds. If this condition is not fulfilled, the $\mathcal{H}_{\infty}$ observer design is impossible. If it is, the solutions for $\Omega$ are given in the following form :

$$
\Omega\left(Y_{\Omega}\right)=\Psi \Theta^{+}+Y_{\Omega}\left(\mathcal{I}_{2 n+m}-\Theta \Theta^{+}\right)
$$

where $Y_{\Omega}$ is a free matrix of appropriate dimension that will be determined later, during the synthesis.

Then, the equality $N=P A-K C$ holds, and the system (26) can be rewritten as

$$
\begin{gathered}
\dot{e}=\Omega\left(Y_{\Omega}\right) \Psi_{1} \quad e+\Omega\left(Y_{\Omega}\right) \Psi_{2} \quad v \\
\text { where } \Psi_{1}=\left[\begin{array}{l}
\mathcal{I}_{n} \\
\mathcal{O}_{n} \\
\mathcal{O}_{m, n} \\
\mathcal{O}_{m, n}
\end{array}\right], \Psi_{2}=\left[\begin{array}{r}
\mathcal{O}_{n, d} \\
D_{x} \\
-D_{y} \\
\mathcal{O}_{m, d}
\end{array}\right] \text { and } \Omega\left(Y_{\Omega}\right)=\Psi \Theta^{+}+Y_{\Omega}\left(\mathcal{I}_{2 n+m}-\Theta \Theta^{+}\right) \text {. }
\end{gathered}
$$


The estimation error, governed by the equation (30), is driven by the unknown disturbance $v$. If the exact observer design is not possible and if the rank condition (28) is fulfilled, the disturbance effect can be minimized and an efficient observer computed. The matrices of the observer can be determined by studying the stability and the $\mathcal{H}_{\infty}$-norm bound of the transfer $e \rightarrow v$. This problem is solved below, by minimizing $\gamma_{\infty}$ such that

$$
\|e\|_{2}<\gamma_{\infty} \cdot\|v\|_{2}
$$

is satisfied.

Proposition 3.1. Consider the system model (5) and the observer (6). Given a positive scalar $\gamma_{\infty}$, if there exist $\mathbf{X}=\mathbf{X}^{T} \succ 0$ and $\tilde{\mathbf{Y}}=\mathbf{X Y}$ satisfying

$$
\begin{gathered}
\left(\begin{array}{ccc}
Q \Psi_{1}+\Psi_{1}^{T} Q^{T} & Q \Psi_{2} & \mathcal{I}_{n} \\
* & -\gamma_{\infty} \mathcal{I}_{d} & \mathcal{O}_{d, n} \\
* & * & -\gamma_{\infty} \mathcal{I}_{n}
\end{array}\right) \prec 0 \\
Q=\mathbf{X} \Psi \Theta^{+}+\tilde{\mathbf{Y}}\left(\mathcal{I}_{2 n+m}-\Theta \Theta^{+}\right)
\end{gathered}
$$

then, the observer (6) is an $\mathcal{H}_{\infty}$-observer according to the Definition 3.1, where the disturbance attenuation level $\gamma_{\infty}$ and the observer matrices are given by

$$
\begin{gathered}
{\left[N, P, K, Y_{E}\right]=\Psi \Theta^{+}+Y_{\Omega}\left(\mathcal{I}_{2 n+2 m}-\Theta \Theta^{+}\right)} \\
\text {where } Y_{\Omega}=X^{-1} \tilde{Y}, \Theta=\left[\begin{array}{rr}
-\mathcal{I}_{n} & \mathcal{O}_{n} \\
A & \mathcal{I}_{n} \\
-C & \mathcal{O}_{m, n} \\
\mathcal{O}_{m, n} & -\left(\mathcal{I}_{m}-D_{y} D_{y}^{+}\right) C
\end{array}\right] \text { and } \Psi=\left[\mathcal{O}_{n}, \mathcal{I}_{n}\right] \text {. }
\end{gathered}
$$

Proof 3.1. The Bounded Real Lemma (?) (BRL) applied to the system (26) gives the solution to (31) and leads to the bi-linear matrix inequality (BMI) (35) where $\mathbf{X}=\mathbf{X}^{T} \succ 0$ and $\mathbf{Y}$ are the unknown matrices to be determined. Therefore the full-order stable and disturbance decoupled observer design problem consists in solving 


$$
\left(\begin{array}{ccc}
Q \Psi_{1}+\Psi_{1}^{T} Q^{T} & Q \Psi_{2} & \mathcal{I}_{n} \\
* & -\gamma_{\infty} \mathcal{I}_{d} & \mathcal{O}_{d, n} \\
* & * & -\gamma_{\infty} \mathcal{I}_{n}
\end{array}\right) \prec 0
$$

where the matrix $Q$ is given by

$$
Q=\mathbf{X} \Omega=\mathbf{X} \Psi \Theta^{+}+\mathbf{X Y}\left(\mathcal{I}_{2 n+2 m}-\Theta \Theta^{+}\right)
$$

The matrix inequality (35) is a BMI since $Q$ is bi-linear in $\mathbf{X}$ and $\mathbf{Y}$. Therefore the change of variable $\tilde{\mathbf{Y}}=\mathbf{X Y}$ is introduced to transform the BMI into a solvable LMI where $Q=\mathbf{X} \Psi \Theta^{+}+\tilde{\mathbf{Y}}\left(\mathcal{I}_{2 n+2 m}-\Theta \Theta^{+}\right)$. Solving (35) with (36) leads to find $\mathbf{X}, \tilde{\mathbf{Y}}$ and thereafter $\mathbf{Y}=\mathbf{X}^{-1} \tilde{\mathbf{Y}}$.

Then $\Omega$ can be deduced by using (29), which gives the observer matrices. Finally, the proposed observer is designed so that the stability conditions (8) are satisfied, and the disturbance decoupling conditions (9) are approached by minimizing $\gamma_{\infty}$ subject to (31).

\subsection{Pole placement}

The previous method ensures the stability of the observer and the minimization of the disturbance effect, but the poles of the observer are obtained through the solution of (32) and may be either very high, have high imaginary parts, or be almost unstable. Such poles may render the observer oscillating and sensitive to measurement noises or unstable in practice. In order to avoid such a behavior that may lead to implementation problems and bad estimation performances, a pole placement method using LMI regions has been introduced into the design procedure, according to the method proposed in ?.

The poles of the observer can be placed in the intersection of the regions $\mathcal{D}_{1}, \mathcal{D}_{2}$ and $\mathcal{D}_{3}$ in the complex plane, corresponding respectively to a conical sector center with inner angle $2 \theta$, a left half plane, and a right half plane, as represented in Figure 3 . These regions are defined by the LMI (37), (38) and (39). The conical sector ensures that the poles lying in this region have a damping ratio at least equal to $\cos (\theta)$, which implies moderate imaginary parts. The half planes $\mathcal{D}_{2}, \mathcal{D}_{3}$ ensure that the poles have real parts in $\left[-p_{\max },-p_{\min }\right]$. 


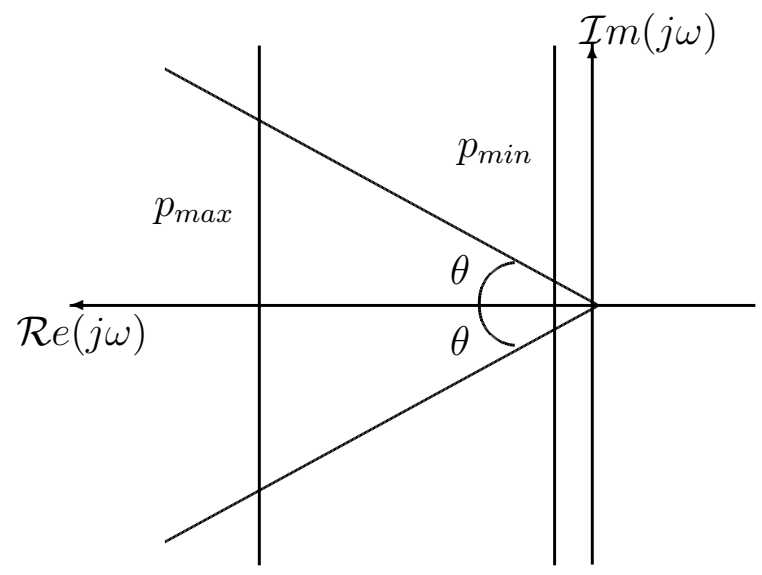

Figure 3: LMI regions in complex plane

$$
\begin{gathered}
\mathcal{D}_{1}=\left\{z \in \mathbb{C}:\left(\begin{array}{cc}
\sin (\theta) \cdot\left(z+z^{*}\right) & \cos (\theta) \cdot\left(z-z^{*}\right) \\
\cos (\theta) \cdot\left(z^{*}-z\right) & \sin (\theta) \cdot\left(z+z^{*}\right)
\end{array}\right) \prec 0\right\} \\
\mathcal{D}_{2}=\left\{z \in \mathbb{C}: z+z^{*}+2 p_{\text {min }} \prec 0\right\} \\
\mathcal{D}_{3}=\left\{z \in \mathbb{C}:-z-z^{*}-2 p_{\max } \prec 0\right\}
\end{gathered}
$$

Proposition 3.2. Consider the system model (5) and the observer (6). Given a positive scalar $\gamma_{\infty}$, if there exist $\mathbf{X}=\mathbf{X}^{T} \succ 0$ and $\tilde{\mathbf{Y}}=\mathbf{X Y}$ satisfying the inequalities

$$
\begin{aligned}
& \left(\begin{array}{ccc}
\mathcal{M}_{11} & \mathcal{M}_{12} & \mathcal{M}_{13} \\
* & \mathcal{M}_{22} & \mathcal{M}_{23} \\
* & * & \mathcal{M}_{33}
\end{array}\right) \prec 0 \\
& \left(\begin{array}{ccc}
Q \Psi_{1}+\Psi_{1}^{T} Q^{T}+2 p_{\min } \mathbf{X} & Q \Psi_{2} & \mathcal{I}_{n} \\
* & -\gamma_{\infty} \mathcal{I}_{d} & \mathcal{O}_{d, n} \\
* & * & -\gamma_{\infty} \mathcal{I}_{n}
\end{array}\right) \prec 0 \\
& \left(\begin{array}{ccc}
-Q \Psi_{1}-\Psi_{1}^{T} Q^{T}-2 p_{\max } \mathbf{X} & Q \Psi_{2} & -\mathcal{I}_{n} \\
* & -\gamma_{\infty} \mathcal{I}_{d} & \mathcal{O}_{d, n} \\
* & * & -\gamma_{\infty} \mathcal{I}_{n}
\end{array}\right) \prec 0
\end{aligned}
$$


where

$$
\begin{aligned}
& \mathcal{M}_{11}=\left(\begin{array}{cc}
\sin (\theta)\left(Q \Psi_{1}+\Psi_{1}^{T} Q^{T}\right) & \cos (\theta)\left(Q \Psi_{1}-\Psi_{1}^{T} Q^{T}\right) \\
-\cos (\theta)\left(Q \Psi_{1}-\Psi_{1}^{T} Q^{T}\right) & \sin (\theta)\left(Q \Psi_{1}+\Psi_{1}^{T} Q^{T}\right)
\end{array}\right) \\
& \mathcal{M}_{12}=\left(\begin{array}{ll}
Q \Psi_{2} & \mathcal{O}_{n, d} \\
\mathcal{O}_{n, d} & Q \Psi_{2}
\end{array}\right) \\
& \mathcal{M}_{13}=\left(\begin{array}{ll}
\sin (\theta) \mathcal{I}_{n} & -\cos (\theta) \mathcal{I}_{n} \\
\cos (\theta) \mathcal{I}_{n} & \sin (\theta) \mathcal{I}_{n}
\end{array}\right) \\
& \mathcal{M}_{22}=-\gamma_{\infty} \mathcal{I}_{2 d} \\
& \mathcal{M}_{23}=\mathcal{O}_{2 d, 2 n} \\
& \mathcal{M}_{33}=-\gamma_{\infty} \mathcal{I}_{2 n}
\end{aligned}
$$

and $Q=\mathbf{X} \Psi \Theta^{+}+\tilde{\mathbf{Y}}\left(\mathcal{I}_{2 n+2 m}-\Theta \Theta^{+}\right)$and $*$ denotes the symmetric element, then the observer (6) is an $\mathcal{H}_{\infty}$-observer according to the Definition 3.1, with the disturbance attenuation level $\gamma_{\infty}$ and whose poles are located in the intersection of LMI regions $\mathcal{D}_{1}, \mathcal{D}_{2}$ and $\mathcal{D}_{3}$. The observer matrices are then given by

$$
\begin{aligned}
& {\left[N, P, K, Y_{E}\right]=\Psi \Theta^{+}+Y_{\Omega}\left(\mathcal{I}_{2 n+2 m}-\Theta \Theta^{+}\right)} \\
& \text {where } \\
& Y_{\Omega}=X^{-1} \tilde{Y}, \Theta=\left[\begin{array}{rr}
-\mathcal{I}_{n} & \mathcal{O}_{n} \\
A & \mathcal{I}_{n} \\
-C & \mathcal{O}_{m, n} \\
\mathcal{O}_{m, n} & -\left(\mathcal{I}_{m}-D_{y} D_{y}^{+}\right) C
\end{array}\right] \text { and } \Psi=\left[\mathcal{O}_{n}, \mathcal{I}_{n}\right] .
\end{aligned}
$$

Proof 3.2. In ?, the LMI constraint allowing the pole placement in region $\mathcal{D}$ defined by

$$
\mathcal{D}=\left\{z \in \mathcal{C}: L+z M+z^{*} M^{T} \prec 0\right\},
$$

is given by

$$
\left(\begin{array}{c|c|c}
L \otimes X+M \otimes(X A) & M_{1}^{T} \otimes(X B) & M_{2}^{T} \otimes C^{T} \\
+M^{T} \otimes\left(A^{T} X\right) & & \\
\hline * & -\gamma \mathcal{I} & D^{T} \\
\hline * & * & -\gamma \mathcal{I}
\end{array}\right) \prec 0,
$$


where $\otimes$ denotes the Kronecker product and the decomposition of $M$ is $M=M_{1}^{T} M_{2}$.

For the LMI region $\mathcal{D}_{1}, L^{\mathcal{D}_{1}}, M_{1}^{\mathcal{D}_{1}}, M_{2}^{\mathcal{D}_{1}}$ can be expressed as

$$
\left\{\begin{array}{l}
L^{\mathcal{D}_{1}}=\mathcal{O}_{2} \\
M_{1}^{\mathcal{D}_{1}}=\mathcal{I}_{2} \\
M_{2}^{\mathcal{D}_{1}}=\left(\begin{array}{cc}
\sin (\theta) & -\cos (\theta) \\
\cos (\theta) & \sin (\theta)
\end{array}\right) .
\end{array}\right.
$$

For the LMI region $\mathcal{D}_{2}, L^{\mathcal{D}_{2}}, M_{1}^{\mathcal{D}_{2}}, M_{2}^{\mathcal{D}_{2}}$ can be expressed as

$$
\left\{\begin{array}{l}
L^{\mathcal{D}_{2}}=2 p_{\text {min }} \\
M_{1}^{\mathcal{D}_{2}}=1 \\
M_{2}^{\mathcal{D}_{2}}=1 .
\end{array}\right.
$$

For the LMI region $\mathcal{D}_{3}, L^{\mathcal{D}_{3}}, M_{1}^{\mathcal{D}_{3}}, M_{2}^{\mathcal{D}_{3}}$ can be expressed as

$$
\left\{\begin{array}{l}
L^{\mathcal{D}_{3}}=-2 p_{\max } \\
M_{1}^{\mathcal{D}_{3}}=-1 \\
M_{2}^{\mathcal{D}_{3}}=-1 .
\end{array}\right.
$$

Then for each region, the constraints can be expressed using (45) with (46), (47) and (48). Therefore three BMIs are obtained, that can be easily transformed into LMIs (40), (41) and (42) using the change of variable $\tilde{\mathbf{Y}}=\mathbf{X Y}$. Then the solvable LMIs (40), (41) and (42) are obtained, where the unknown matrices are $\tilde{\mathbf{Y}}$ and $\mathbf{X}=\mathbf{X}^{T} \succ 0$.

\subsection{Design methodology}

The procedure to design the $\mathcal{H}_{\infty}$-observer is represented in Figure 4 and can be summarized as follows:

Step 1: Determine the region where the poles of the observer have to be located, and choose the pole bounds $p_{\min }, p_{\max }$ and the cone angle $\theta$.

$\underline{\text { If } D_{y}=0:}$

Step 2a: Check the exact disturbance decoupling condition (13). 
- If (13) is fulfilled, find $E$ according to (14), such that $(P A, C)$ is detectable, if possible.

- If (13) is not fulfilled, the exact disturbance decoupling is impossible, but an approximated observer may be found. Go to Step 4.

\section{Step 3a:}

- If $(P A, C)$ is detectable, place the poles using $K$, if possible. Then deduce the observer matrices $N=P A-K C, L=K-N E$ and $E$. Check the poles of the computed exact observer.

- If $(P A, C)$ is not detectable, the observer design is impossible.

$\underline{\text { If } D_{y} \neq 0:}$

Step 2b: Check the exact observer existence conditions (19).

- If (19) is fulfilled, find $Y_{L Y E}$ such that (22) is fulfilled and $\left((P A-L C) P^{+},\left(\mathcal{I}_{n}-\right.\right.$ $\left.P P^{+}\right)$) is detectable, if possible. Then deduce the observer matrices $L$ and $E$.

- If (19) is not fulfilled, the exact disturbance decoupling is impossible. Go to Step 4.

\section{Step 3b:}

- If (22) is fulfilled and $\left((P A-L C) P^{+},\left(\mathcal{I}_{n}-P P^{+}\right)\right)$is detectable, place the poles of $N$ using $Y_{N}$, if possible. Check the poles of the computed exact observer.

- If (22) is not fulfilled, or $\left((P A-L C) P^{+},\left(\mathcal{I}_{n}-P P^{+}\right)\right)$is not detectable, an approximated observer may exist. Go to Step 4.

If exact decoupling is not possible or if the poles cannot be placed in the desired region, then a trade-off can be found between pole placement and disturbance decoupling according to the following procedure: 


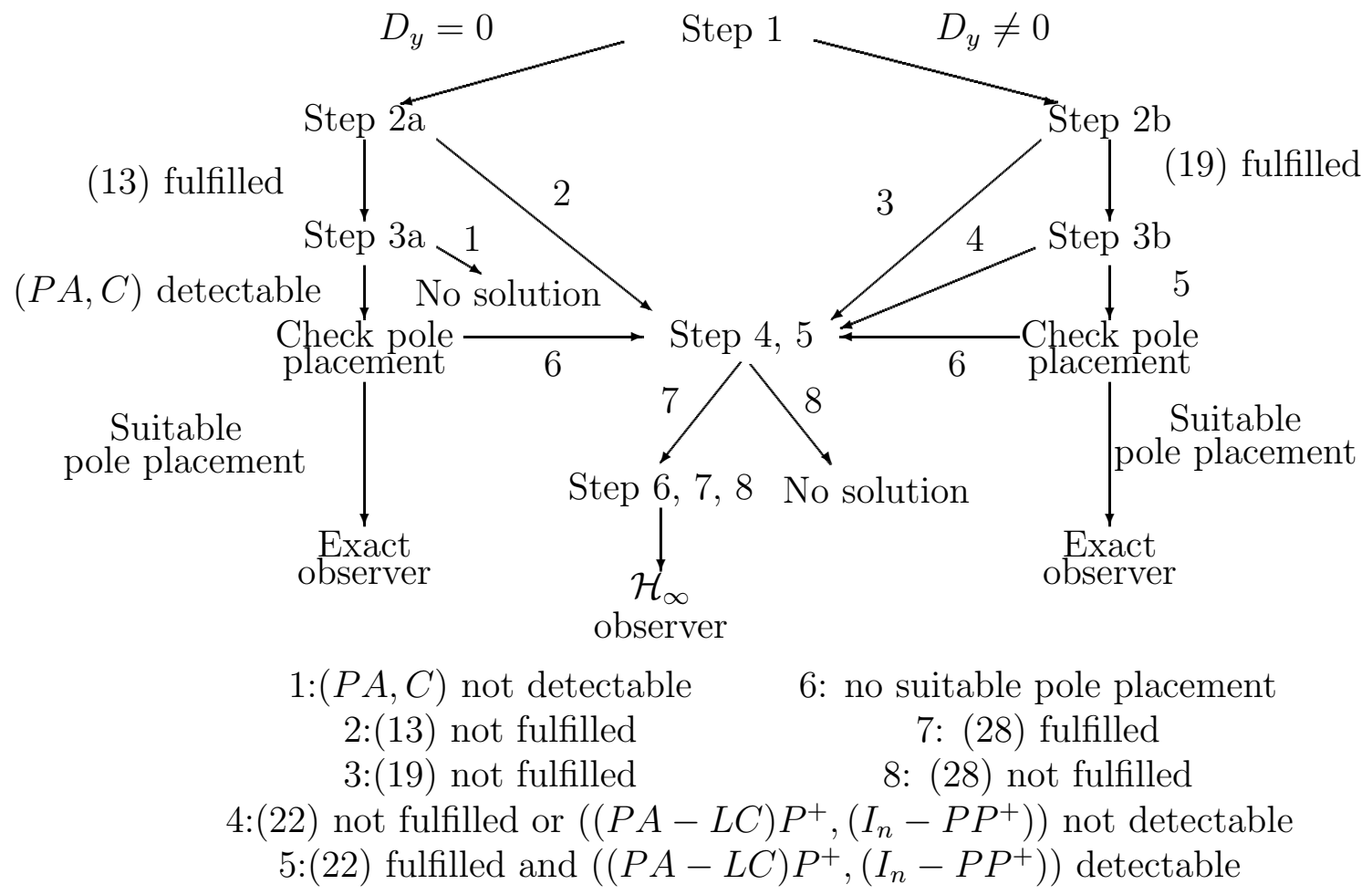

Figure 4: Observer design procedure

Step 4: If the rank condition (28), is fulfilled, go to Step 5. If not, the $\mathcal{H}_{\infty}$-observer design is impossible.

Step 5: Minimize $\gamma_{\infty}$ under LMI (40), (41) and (42).

Step 6: Calculate $Y_{\Omega}=X^{-1} \tilde{Y}$, and $\Omega$ using (29).

Step 7: Deduce the observer matrices $N$ from $\Omega, E$ from $P=\mathcal{I}+E C$ and $L$ from $K=L+N E$.

pdf

\section{Synthesis results and robustness analysis}

pdf Some numerical synthesis results are given in Section 4.1, and a robust analysis is performed in Section 4.2.

\subsection{Numerical synthesis results}

In this paragraph, the procedure described in Section 3.5 is applied to design the observer. 
Figure 5: Poles of the observer and specified regions

Step 1: The LMI regions (37), (38) and (39) have been chosen and are determined by

$$
\left\{\begin{array}{ccc}
\theta & = & \frac{\pi}{4} \\
p_{\min } & = & 1 \\
p_{\max } & = & 200
\end{array} .\right.
$$

This region is represented in Figure 5.

Steps 2b: Here, the measured unsprung mass accelerations depend on the ground disturbance, thereafter $D_{y} \neq 0$. Unfortunately, (19) is not fulfilled. The exact disturbance decoupling is not possible. Therefore, the approximated observer design approach has been chosen to tackle both the disturbance decoupling and the pole placement problems. Step 4: The rank condition (28) is fulfilled.

Step 5: The minimal $\gamma_{\infty}$ obtained solving the LMI problem (40-42) is $\gamma_{\infty}=1.08$.

Steps 6-7: The matrices of the observer have been deduced and the poles of the observer are located in the specified region, represented in Figure 5.

Remark 4.1. The pole bounds $p_{\min }$ and $p_{\max }$ have to be chosen by the designer. The lower bound of the poles must be chosen according to the noise level. If there is almost no measurement noise, this bound can be set equal to 0 so that pure integrators are allowed. Therefore, the results will be of course more accurate. But if the noise level is higher, the observer may become unstable due to very small poles and the lower bound must be increased. Therefore, a well-known compromise has to be found between performance and robustness, using this simple adjustment parameter. In the case of the experiment described in Section 5, the observer becomes unstable if $p_{\min }<1$, since the measurement noise is important. The upper bound has also to be chosen by the designer. It determines the bandwidth of the observer. The observer must be at least, ten times faster than the system to be observed to get accurate results. However, for noise filtering, this bound has to be less than the frequencies of the noise. For the application considered in this paper, 
Figure 6: Transfer $\|e / v\|$ - Bode diagrams

the noise is located in the frequency range $[800-1200 H z]$. Therefore $p_{\max }=200$ allows the observer to filter the noise.

In order to analyze the achieved disturbance decoupling, the Bode diagrams of the transfer functions between the ground disturbance $v$ and the estimation error $e$ on each state have been computed for the 14 state variables. Some of them, corresponding to the analysis of the front left suspension, are given on Figure 6. These results emphasize the attenuation of the ground disturbance effect on the estimation error, since the largest disturbance amplification of the 14 errors, over the whole frequency range is $-60 \mathrm{~dB}$.

\subsection{Robustness analysis}

In this section, the closed-loop system $\Sigma(s)$, including the vehicle model $V(s)$ given by the Equation (5) and the observer $O(s)$ given in (6), is considered. The state estimation error $e=x-\hat{x}$ is used as the "closed-loop" system output for robustness analysis. The robustness of the designed $\mathcal{H}_{\infty}$-observer is studied using the $\mu$-analysis tool. The considered parametric uncertainties, defining the uncertain system, are given in Table 3 .

\begin{tabular}{lll}
\hline Uncertain parameter & Variation & \\
\hline \hline$m_{s}$ & 1450 & $\pm 50 \%[\mathrm{~kg}]$ \\
$m_{u s_{i}}, i=1 . .4$ & 39 & $\pm 10 \%[\mathrm{~kg}]$ \\
$k_{i}, i=1 . .4$ & 30000 & $\pm 20 \%[\mathrm{~N} / \mathrm{m}]$ \\
$c_{i}, i=1 . .4$ & 500 to & $6000[\mathrm{Ns} / \mathrm{m}]$ \\
$k_{t i}, i=1 . .4$ & 18000 & $\pm 30 \%[\mathrm{~N} / \mathrm{m}]$ \\
$I_{x}$ & 610 & $\pm 30 \%[\mathrm{~N} * \mathrm{~m} / \mathrm{rad}]$ \\
$I_{y}$ & 2750 & $\pm 30 \%\left[\mathrm{~N}^{*} \mathrm{~m} / \mathrm{rad}\right]$ \\
\hline
\end{tabular}

Table 3: Parameter uncertainties 
The main uncertainties concern the sprung mass and the damping rates. The sprung mass depends on the number and weight of passengers, quantity of fuel etc... and the damping rate depends on the control signal if the shock absorber is controlled. It also depends on the nonlinearities of the shock absorber, and therefore on the shock absorber technology. SOBEN shock absorber is nonlinear and has a controlled damping rate varying from 500 to $6000 \mathrm{Ns} / \mathrm{m}$. In the synthesis of the observer, a mean damping rate value has been used, but the robustness with respect to this parameter variation is very important when the observer is used in a suspension control application.

These parametric uncertainties have been considered and used to perform a classical $\mu$-analysis in order to study both robust stability and performances (?), where $\mu$ denotes the structured singular value.

\section{Robust stability:}

The considered uncertain system for robust stability analysis is presented in Figure 7 using the classical LFT form, where $\Delta_{r}(s)$ represents the structured uncertainties corresponding to the parametric uncertainties given in Table 3. $\Sigma(s)$ is the closed-loop system (7) including both the system to be observed and the observer. The robust stability is ensured if and only if the closed-loop system is stable and if the inequality

$$
\mu_{\Delta_{r}(L(s))}<1, \quad \forall s=j \omega
$$

is fulfilled. The structured singular value $\mu$ cannot be exactly computed. However, a numerical algorithm has been used to compute the upper and lower bounds of $\mu$. These results given in Figure 8 show that $\mu$ is less than 1 and does not depend on the frequency. Therefore the system remains stable, whatever the parameters.

\section{Robust performance:}

The considered uncertain system for robust performance analysis is presented in Figure 9. This system is similar to the one used for robust stability analysis, but some fictitious uncertainties $\Delta_{f}(s)$ representing the performance objectives have been added. They are 


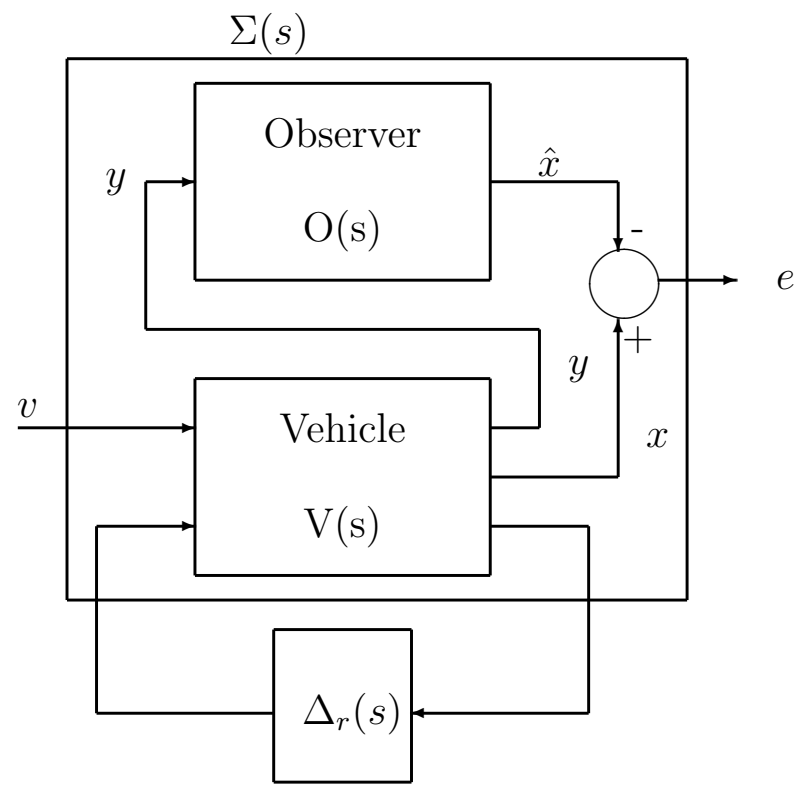

Figure 7: Uncertain system for robust stability analysis

Figure 8: Upper and lower bounds of $\mu$ for robust stability 


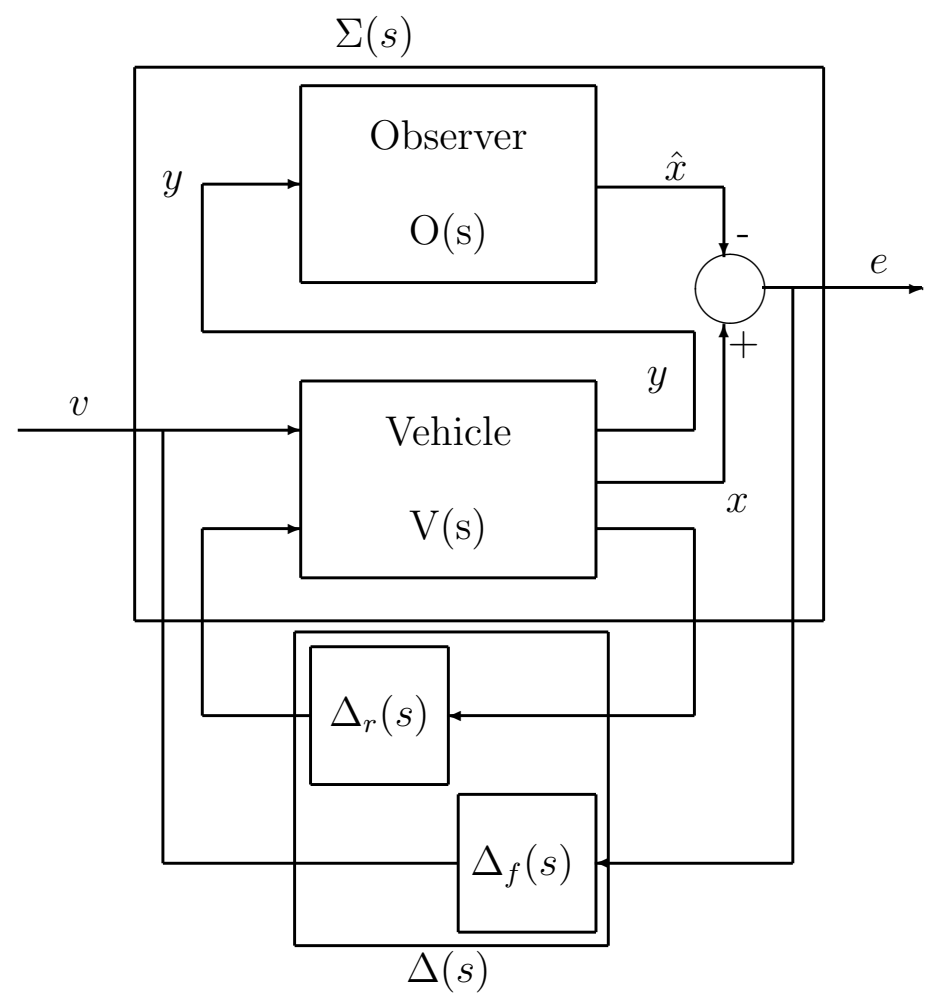

Figure 9: Uncertain system for robust performance analysis

given by the low-pass filter

$$
W(s)=G \cdot \frac{2 \pi f_{c}}{s+2 \pi f_{c}}
$$

where $f_{c}=20 \mathrm{~Hz}$ denotes its cut-off frequency and $G=1$ its static gain. This weighting filter focuses the performances objectives on the bandwidth of the system to be observed. Then, using this new uncertain system including the performance objectives, the robust performance is ensured if and only if the closed-loop system is stable and if

$$
\mu_{\Delta}(\Sigma(s))<1, \quad \forall s=j \omega
$$

is fulfilled, where $\Delta(s)$ is given by

$$
\Delta(s)=\left(\begin{array}{ll}
\Delta_{r}(s) & \mathcal{O} \\
\mathcal{O} & \Delta_{f}(s)
\end{array}\right)
$$

and includes both the real structured uncertainties and the performance objectives.

The upper and lower bounds of $\mu$ have also been computed for the system given in 
Figure 10: Upper and lower bounds of $\mu$ for robust performance

Figure 9. These results presented in Figure 10 show that $\mu$ is always less than 1 . The performances are ensured, whatever the parameter uncertainties.

These results emphasize the $\mathcal{H}_{\infty}$-observer robustness. The designed observer is not sensitive to the studied parametric uncertainties including all the possible parameter variations of a real vehicle equipped with controlled shock absorbers. pdf

\section{Experimental results}

pdf

\subsection{Description and set-up of the experiment}

Four semi active shock absorber prototypes have been built by SOBEN and mounted on a test car, as represented in Figure 1. Each shock absorber can be controlled by a servomechanism. This actuator allows to control the damping rate of each suspension in real-time. The observer proposed in this paper is to be used in a suspension control strategy, but the experiment presented here has been run to test the observer performances only. Therefore, no control strategy was implemented. The influence of the damping rate on the observer performance has already been discussed in the robust analysis presented in Section 4.2 and the observer is robust to damping rate variations from 500 to $6000 \mathrm{Ns} / \mathrm{m}$. In order to confirm this result, several experiments have been run with the observer proposed here, for different varying control signals (sinusoidal signal varying between a minimal value and a maximal value), which corresponds to varying damping rates. The damping rate does not influence the accuracy of the estimations. This shows that taking these variations into account in the observer design is not useful.

The data acquisition is done by a set of five electronic boards developed by SOBEN. Each shock absorber has a small acquisition board, represented in Figure 18, that converts the analog measurements into CAN (Controller Area Network) frames. A central board where the observer is implemented receives the frames through the CAN network and computes the estimated states for each shock absorber, using the four unsprung masses 
accelerations and the three sprung mass accelerations. This architecture is described in Figure 18. The four shock absorber boards are operational, but the central board has not been programmed yet. Therefore a Dspace board has been used for the experiments presented in this paper. The implementation of the observer is done automatically by the software provided by Dspace. However, the implementation cannot be done if the observer is ill-conditioned. Therefore, the poles of the observer have to be properly chosen.

\subsection{Estimation results}

The experimental results obtained here with the observer designed in Section 3 are given. The observer has been tested while the car was traveling at $70 \mathrm{~km} / \mathrm{h}$ on a bad mountain road. The shock absorber control signals were constant and nominal. During the experiment, the following variables have been estimated and measured:

- The four unsprung masses vertical accelerations $\left(\ddot{z}_{u s_{i}}\right.$, see Figure 11)

- The three sprung mass vertical accelerations $\left(\ddot{z}_{s_{i}}\right.$, see Figure 12)

- Deflection velocities $\left(\dot{z}_{\text {defi }}\right.$, see Figure 13)

- Deflections $\left(z_{\text {def }}\right.$, see Figure 14$)$

- Vertical acceleration of the center of gravity $\left(\ddot{z}_{s}\right.$, see Figure 17)

- Roll and pitch angular velocity $(\dot{\theta}, \dot{\phi}$, see Figure 15)

- The four unsprung masses vertical velocities $\left(\dot{z}_{u s_{i}}\right.$, see Figure 16)

The measured deflection velocities have been derived from the measured deflections whereas the measured unsprung masses velocities have been integrated from the measured accelerations.

Figures 11 and 12 show the measured and estimated sprung and unsprung masses accelerations for suspensions 1 and 4. The other ones have not been represented but the results are similar. These variables correspond to the measurements used by the 
$\mathcal{H}_{\infty}$-observer. The relative errors given by Table 4 and computed using the present experimental results are less than 1\%. Therefore, the estimated variables are very similar to the measurements, which is quite normal since these measurements are used by the observer.

Figures 13 and 14 represent the measured and estimated deflection velocities as well as the measured and estimated deflections for suspensions 1 and 4. These results emphasize the observer performance and accuracy for the $\mathcal{H}_{\infty}$-observer since the variables have been estimated and filtered in real-time without any delay. The relative errors of the estimated deflection velocities, given in Table 4 are less than 1\%, which is very accurate, whereas the deflections have relative errors in the region of 15\%. The estimated deflections are not as accurate as for the deflection velocities since the observer does not contain pure integrators. The absolute value of the minimal real part of the poles has been indeed constrained to be greater than 1 for stability reasons. However, if the measurement noise could be reduced, smaller minimal poles magnitudes could be allowed, and the estimated positions would be more accurate.

The deflection velocities are very important since many control strategies developed in the past few years are based on these measurements (????). Furthermore, the deflection sensor is very expensive and has a short life-time. The results obtained on this test car show that the estimated deflection velocities are satisfactory and can be used to control the shock absorber.

The results given in Figures 15 and 16 concern the estimation of the unsprung masses velocities and of the angular pitch and roll velocities. These figures show that the estimated variables are very similar to the measured ones. The relative errors and variances given in Table 4 are less than $0.2 \%$ for the unsprung masses velocities, and less than $2 \%$ for the angular velocities, therefore these estimated variables can be used in a control strategy to improve the vehicle behavior.

It should also be noticed that the measurements of the angular velocities, provided by the gyrometers are very noisy. The results show that the estimated variables are not 


\begin{tabular}{cccc}
\hline Figure & Variable & MRE $^{1}[\%]$ & RV $^{2}[\%]$ \\
\hline \hline 11 & $\ddot{z}_{u s_{1}}$ & 0.14 & 0.022 \\
11 & $\ddot{z}_{u s_{4}}$ & 0.11 & 0.028 \\
12 & $\ddot{z}_{s_{1}}$ & 0.42 & 0.004 \\
12 & $\ddot{z}_{s_{4}}$ & 0.84 & 0.007 \\
13 & $\dot{z}_{\text {def }}$ & 0.44 & 1.054 \\
13 & $\dot{z}_{\text {def }}$ & 0.73 & 2.065 \\
14 & $z_{\text {def }}$ & 14.8 & 10.29 \\
14 & $z_{\text {def }}$ & 17.4 & 9.045 \\
17 & $\ddot{z}_{s}$ & 3.80 & 0.769 \\
15 & $\dot{\phi}_{15}$ & 1.76 & 19.75 \\
15 & $\dot{\theta}$ & 2.01 & 14.87 \\
16 & $\dot{z}_{u s_{1}}$ & 0.11 & 0.185 \\
16 & $\dot{z}_{u_{4}}$ & 0.19 & 0.236 \\
& ${ }^{1}{ }_{\text {Mean Relative Error }}{ }^{2}$ Relative Variance
\end{tabular}

Table 4: Experimental results: relative mean errors and variances 
corrupted by this noise. Therefore the bandwidth of the observer is appropriate, thanks to the pole placement method proposed in Section 3.4.

The variances given in Table 4 are always less than 5\%, except for the deflections and angular velocities. This is due to the important measurement noise that corrupts these measured variables, but the estimated variables are not corrupted by this noise.

Figure 11: Unsprung mass 1 (top) and 4 (bottom) vertical acceleration

Figure 12: Sprung mass 1 (top) and 4 (bottom) vertical acceleration

Figure 13: Deflection velocity 1 (top) and 4 (bottom)

pdf

\section{Conclusion}

pdf In this paper, a methodology to design an unknown input observer for a suspension control application has been developed. This observer, based on reliable and cheap accelerometers, provides a good estimation of the sprung and unsprung mass vertical ac-

celerations. The estimation is decoupled from the unknown road disturbance through an $\mathcal{H}_{\infty}$ minimization. The proposed synthesis method also includes a pole placement in LMI regions to avoid ill-adapted dynamics that may preclude the implementation and damage the estimation accuracy in the real embedded application. Therefore, the procedure presented in this paper is a complete and practical observer design procedure for automotive 
Figure 14: Deflection 1 (top) and 4 (bottom)

Figure 15: Roll (top) and pitch (bottom) angular velocity

suspension control applications. This observer has been implemented and embedded on a test car. The experimental results emphasize the observer performances and robustness. Furthermore, the tuning of the observer is simple since only the model and the desired bandwidth of the observer have to be known. It is worth noting that, even if the observer is a LTI system, it has been proven to be efficient with respect to the variation of the damping coefficient of the suspension, which, in practice, corresponds to different control actions (for the semi-active damper).

Future works will consist in designing a reduced-order observer. The observer could also be scheduled according to the damping rate $c$, even if this does not seem to be necessary for the considered application. pdf

\section{Appendix}

pdf In the appendix, the complete acquisition and control board/arcitecture is detailed.

The vehicle is equipped with various sensors for each suspension. The acquisition of these data is done by a set of five electronic boards developed by SOBEN. Each shock absorber is equipped with a small acquisition board, represented in Figure 18, that converts the analog measurements into CAN (Controller Area Network) frames. A central board where an observer is implemented, receives the frames through the CAN network, computes on-line the control signal of each shock absorber and sends it to the four shock

Figure 16: Unsprung mass 1 (top) and 4 (bottom) vertical velocity 
Figure 17: Sprung mass center of gravity vertical acceleration

absorber control boards through the CAN network. This architecture is described in Figure 18.

Figure 18: Shock absorber control board (left) and complete control architecture (right)

The four shock absorber boards are operational but the central board has not been programmed yet. Therefore a Dspace card has been used for the experiments presented in this paper. The implementation of the controllers is done automatically by the software provided by Dspace, from a Matlab-Simulink program. A PC can be connected to analyze and record on-line the different signals.

pdf

\section{References}

Ben-Israel, A., Greville, T., 2003. Generalized inverses. Springer-Verlag.

Chilali, M., Gahinet, P., Apkarian, P., 1999. Robust pole placement in LMI regions. IEEE Transactions on Automatic Control 44 (12), 2257-2270.

Darouach, M., 2000. Existence and design of functional observers for linear systems. IEEE Transactions on Automatic Control 45 (5), 940-943.

Darouach, M., Zasadzinski, M., Xu, S., 1994. Full-order observers for linear systems with unknown inputs. IEEE Transactions on Automatic Control 39 (3), 606-609.

Elbeheiry, E. M., Karnopp, D. C., Elaraby, M. E., Abdelraaouf, A. M., 1996. Suboptimal design of active and passive suspensions based on a full car model. Vehicle System Dynamics 26, 197-222.

Esmailzadeh, E., Fahimi, F., 1997. Optimal adaptive active suspensions for a full car model. Vehicle System Dynamics 27, 89-107. 
Hedrick, J., Rajamani, R., Yi, K., 1994. Observer design for electronic suspension applications. Vehicle System Dynamics 23 (6), 413-440.

Hou, M., Muller, P. C., june 1994. Disturbance decoupled observer design: A unified viewpoint. IEEE Transactions on Automatic Control 32 (6), 1338-1341.

Hsu, L., Chen, T., 2009. Vehicle full-state estimation and prediction system using state observers. IEEE Transactions on Vehicular Technology 58 (6), 2651-2662.

Koenig, D., 2006. Observer design for unknown input nonlinear descriptor systems via convex optimization. IEEE Transactions on Automatic Control 51 (6), 1047-1052.

Koenig, D., Marx, B., Jacquet, D., 2008. Unknown input observers for switched nonlinear discrete time descriptor systems. IEEE Transactions on Automatic Control 53 (1), $373-379$.

Poussot-Vassal, C., Sename, O., Dugard, L., Gaspar, P., Szabo, Z., Bokor, J., december 2008. A new semi-active suspension control strategy through LPV technique. Control Engineering Practice 16 (12), 1519-1534.

Rajamani, R., Hedrick, J., 1995. Adaptive observers for active automotive suspensions: theory and experiment. IEEE Transactions on Control Systems Technology 3 (1), 8693.

Sammier, D., 2001. Sur la modélisation et la commande de suspensions de véhicules automobiles. Phd thesis, Institut National Polytechnique de Grenoble.

Sammier, D., Sename, O., Dugard, L., april 2003. Skyhook and $\mathcal{H}_{\infty}$ control of semi-active vehicle suspensions: some practical aspects. Vehicle System Dynamics 39 (4), 279-308.

Savaresi, S. M., Siciliani, E., Bittanti, S., 2005. Acceleration driven damper: an optimal control algorithm for comfort oriented semi-active suspensions. ASME Transactions: Journal of Dynamic Systems, Measurements and Control 127 (2), 218-229.

Scherer, C., Weiland, S., 1999. LMI in control (lecture support, DELFT University), www.dcsc.tudelft.nl/ cscherer/lmi.html. 
Skogestad, S., Postlethwaite, I., 2005. Multivariable Feedback Control: Analysis and Design. John Wiley and Sons.

Spelta, C., 2008. Design and applications of semi-active suspension control systems. Phd thesis, Politecnico di Milano, dipartimento di Elettronica e Informazione, Milano, Italy.

Tsui, C. C., 1996. A new design approach to unknown input observers. IEEE Transactions on Automatic Control 41 (3), 464-468.

Valcher, M., 2000. State observer for discrete-time linear systems with unknown inputs. IEEE Transactions on Automatic Control 44 (2), 397-401.

Yi, K., 1995. Design of disturbance decoupled bilinear observers. KSME Journal 9 (3), $344-350$.

Yi, K., Song, B. S., 1999. Observer design for semi-active suspension control. Vehicle System Dynamics $32(2-3)$.

Zin, A., 2005. Sur la commande robuste de suspensions automobiles en vue du contrle global de chassis. Phd thesis, Institut National Polytechnique de Grenoble, Grenoble, France. 